\section{Ambiente educativo en una facultad de medicina peruana: una tendencia negativa a lo largo de los años académicos.}

\author{
OSCAR FLORES-FLORES ${ }^{\mathrm{a}}$, YASMÍN LAJO-AURAZO ${ }^{\mathrm{b}}$, \\ ALEJANDRO ZEVALLOS-MORALES ${ }^{b}$, PAOLA L. RONDÁN ${ }^{c}$, \\ FRANK LIZARASO-SOTO $^{\mathrm{d}}$, TAMARA JORQUIERA ${ }^{\mathrm{e}}$
}

\section{Educational environment in a Peruvian medical school. A negative trend over the academic years}

Background: Educational environment refers to the material resources and interpersonal relationships of an educational institution. Aim: To describe the educational environment of a Peruvian medical school and to explore a possible association between curricular years and the educational environment. Material and Methods: A cross-sectional study was conducted using the Dundee Ready Education Environment Measure (DREEM) to evaluate the educational environment of a Peruvian medical school. Data collection consisted in online surveys completed voluntarily by $1^{\text {st }}$ through $6^{\text {th }}$ year medical students between April and October 2015. Results: The questionnaire was completed by 828 of $2,421(34.2 \%)$ students. The mean DREEM score was $117 \pm 25.6$ of a maximum of 200. A poorer perception of the educational environment was associated with later years in the curriculum, when analysis was adjusted for gender, age and academic status $(p<0.001)$. Conclusions: The educational environment of this medical school scored positively (> 100 points). However, it was evident that medical students in later curricular years had a more negative perception of the educational environment compared to those in earlier academic years.

(Rev Med Chile 2018; 146: 53-63)

Key words: Education; Education, Medical; Education, Medical, Undergraduate; Peru; Teaching.

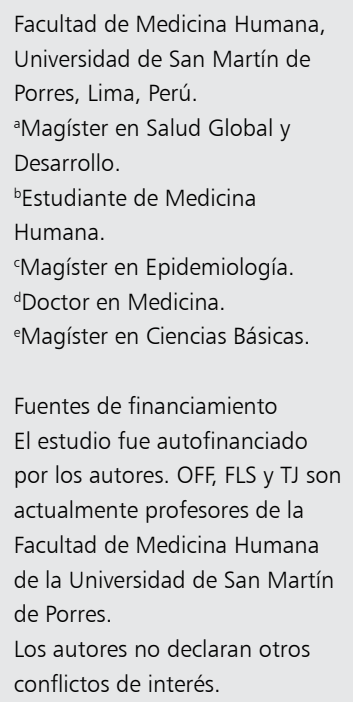

Recibido el 10 de junio de 2017, aceptado el 15 diciembre de 2017.

Correspondencia a:

Dr. Oscar Flores-Flores

Facultad de Medicina Humana,

Universidad de San Martín de Porres.

Av. Alameda del Corregidor 1531, Urb. Los Sirius, Las Viñas, La Molina, Lima 12.

Teléfono: (+511) 934901717

ofloresf@usmp.pe
$\mathrm{E}$ l ambiente educativo es un constructo amplio que hace referencia a las condiciones materiales e intangibles de una institución, incluyendo las relaciones entre las personas y las características de estas interacciones ${ }^{1}$. El ambiente educativo influencia en gran parte el aprendizaje en las facultades de medicina, y se encuentra asociado al éxito y la satisfacción de los estudiantes ${ }^{2,3}$. La percepción que los estudiantes tengan sobre el ambiente educativo provee una base para la comprensión y mejora de los elementos que lo conforman, para así encaminarla hacia los objetivos institucionales ${ }^{4}$.

A lo largo de los años se han ido desarrollando diversos instrumentos ${ }^{5-7}$ para poder medir el ambiente educativo en las universidades. Entre ellos, el más usado actualmente es el Dundee Ready Educational Environment Measure (DREEM) $)^{8}$, un cuestionario creado para evaluar el ambiente educativo en facultades afines a las ciencias de la salud. El cuestionario DREEM ha sido traducido tanto al español como a otros idiomas ${ }^{9}$ y se ha aseverado 
que es un instrumento confiable, además de ser culturalmente independiente ${ }^{10}$.

En Latinoamérica, escuelas de medicina de Argentina y Chile han utilizado el instrumento DREEM $^{11,12}$. En un estudio, se comparó el puntaje entre una universidad de Chile y otra de Argentina, encontrándose una mejor evaluación en esta última, atribuido a su currículo basado en problemas $^{11}$. En otro estudio, se utilizó el DREEM para comparar 6 facultades de medicina, identificando áreas que requerían cambios ${ }^{2}$. En el 2016, se publicó un estudio que comparó una facultad chilena con otra española, mostrando que en ambas, la percepción educativa disminuyó en alumnos de años superiores ${ }^{13}$.

En Perú, se ha evidenciado el progresivo aumento de facultades de medicina de calidad cuestionable $^{14}$. Por otro lado, se ha creado recientemente la Superintendencia Nacional de Educación Superior Universitaria (SUNEDU), cuyo objetivo es evaluar y asegurar la calidad de educación en las universidades, incluyendo las facultades de medicina ${ }^{15}$. Sin embargo, a pesar del creciente reconocimiento de la importancia del ambiente educativo, no se han encontrado estudios que examinen el ambiente educativo de una facultad de medicina peruana.

El objetivo de este estudio fue describir el ambiente educativo de una facultad de medicina peruana de una universidad privada, y determinar si existía asociación entre el año académico y la percepción del ambiente educativo de esta facultad médica.

\section{Materiales y Métodos}

\section{Diseño del estudio}

Se condujo un estudio transversal usando un cuestionario anónimo virtual. Se accedía a este a través del aula virtual de la facultad de medicina, plataforma que los estudiantes usan para obtener material de lectura, entregar trabajos o rendir exámenes.

\section{Áreas $y$ participantes}

La Facultad de Medicina de la Universidad de San Martín de Porres (USMP), una institución privada reconocida en el Perú, ha iniciado una reforma interna para adecuarse a los estándares de calidad que exige la SUNEDU ${ }^{16}$.
En la USMP, el programa de pregrado es similar a la mayoría de facultades de medicina peruanas y latinoamericanas, dividida en 3 etapas: preclínica, clínica e internado. Durante la etapa preclínica (los primeros tres años), los estudiantes permanecen principalmente en el campus universitario, aprenden los fundamentos básicos de la medicina, y su entrenamiento está compuesto por clases teóricas, prácticas de laboratorio y seminarios. Durante la etapa clínica (los siguientes tres años), los estudiantes reciben entrenamiento en ambientes hospitalarios y clases teóricas. La etapa final es el internado (último año), que permite a los estudiantes adquirir experiencia preprofesional al convertirse en fuerza de trabajo del hospital bajo la supervisión de médicos asistentes.

La población objetivo de este estudio consistió en estudiantes de medicina de $1^{\circ}$ a $6^{\circ}$ año académico de la USMP, sede Lima. El $7^{\circ}$ año (internado) no fue incluido, debido a que estos estudiantes no utilizan regularmente el aula virtual. Los datos fueron recolectados entre abril y octubre del 2015. Solo se consideraron en al análisis final los cuestionarios completamente llenados.

\section{Definición de las variables}

El ambiente educativo fue medido usando el cuestionario DREEM validado al español y utilizado extensivamente en Chile $^{12}$, que consiste de 50 ítems y 5 dominios: percepción de aprendizaje, percepción de los docentes, autopercepción académica, percepción de la atmósfera, y autopercepción social. Para interpretar los puntajes, los autores originales del DREEM ${ }^{17}$ recomendaron que toda la escala y cada subescala tengan 4 puntos de corte, esquematizados en la Tabla 2. Además, señalan que los ítems individuales con puntaje $<2,00$ deben ser entendidas como "áreas problema", y aquellas con puntaje $>3,50$ como "áreas fuertes". Sin embargo, algunos estudios han cuestionado la utilidad y validez de las subescalas ${ }^{8,19,20}$. Por eso, aunque nuestro estudio muestra todos los resultados, la discusión se centra principalmente en el puntaje global, la diferencia entre años académicos y la identificación de áreas problemas y áreas fuertes.

El año académico fue medido como una variable continua discreta $\left(1^{\circ}\right.$ a $\left.6^{\circ}\right)$. Las etapas académicas de forma categórica: etapa preclínica (los primeros tres años) y etapa clínica (los siguientes tres años académicos). Otras variables 
incluidas fueron: edad (en años), sexo, condición de matriculado (regular/no regular). La condición de matrícula "no regular" se refiere a aquellos estudiantes que no se matricularon en todos los cursos correspondientes a su año académico, lo cual es generalmente causado por problemas académicos o económicos. Todas las variables fueron autorreportadas.

\section{Procedimientos}

Un piloto con 15 estudiantes de medicina se realizó para identificar cualquier dificultad en entender los ítems del cuestionario. Se realizaron pequeños cambios gramaticales para mejorar el entendimiento, y estos estudiantes no participaron en el estudio final. Todos los estudiantes registrados en la facultad de medicina de $1^{\circ}$ a $6^{\circ}$ año académico fueron invitados a participar mediante el aula virtual. La invitación aparecía como ventana emergente ("pop up"). Además, incluía un formato de consentimiento informado que explicaba el propósito de estudio y el cuestionario DREEM.

\section{Análisis de los datos}

Los datos fueron analizados usando STATA 13 para Windows (STATA Corp, CollegeStation, TX, US). Las características de la muestra fueron descritas de acuerdo a los años académicos. Medias y desviaciones estándar fueron usadas para resumir las variables numéricas. La prueba $t$ de Student fue usada para comparar los puntajes del DREEM entre etapas preclínicas y clínicas. Modelos de regresión lineal simple y ajustado fueron usados para identificar asociaciones del puntaje DREEM y los años académicos, y puntaje DREEM y etapas académicas, usando errores estándar robustos.

\section{Ética}

Aprobación ética fue obtenida del Comité Institucional de Ética en Investigación de la Universidad de San Martín de Porres, en Lima, Perú. El sorteo de un libro de texto de medicina entre los participantes de cada año académico fue incluido para incentivar la participación en el estudio. Solo se solicitó el código de estudiante para el sorteo, para el análisis todos los códigos fueron eliminados para no violar el anonimato de los participantes. Toda participación fue voluntaria. El proceso de consentimiento se llevó a cabo como los recomienda el reglamento institucional de investigación y del comité de ética. Los resul- tados encontrados fueron discutidos con algunas autoridades de la facultad de medicina humana. Los resultados expuestos tienen un enfoque constructivo, son parte de la búsqueda constante de la mejora educativa.

\section{Resultados}

\section{Descripción de la población de estudio}

Un total de 2.421 estudiantes de $1^{\circ}$ a $6^{\circ}$ año estaban registrados en esta facultad de medicina para el año 2015. De estos, $911(37,6 \%)$ empezaron el cuestionario, pero solo $828(32,4 \%)$ lo completaron. Dentro de los encuestados, $489(59,1 \%)$ eran mujeres. La edad promedio fue de 20,6 (DE: 2,5) años. La mayoría de participantes pertenecían al quinto año $(44,3 \%)$ y segundo año $(25,8 \%)$. Hubo $121(14,6 \%)$ estudiantes con matrícula no regular. La distribución de estos estudiantes fue variada en los años académicos, siendo más frecuente en la etapa preclínica (Tabla 1 ).

\section{Puntaje global de la escala DREEM en la muestra}

El puntaje global de la escala DREEM en la muestra fue de 117,2 (DE: 25,6). Este resultado fue mayor al puntaje mínimo (100 puntos) necesario para considerarlo más positivo que negativo ( $\mathrm{Ta}-$ bla 2). En todos los años académicos, el puntaje global fue mayor a 100 puntos, encontrándose el puntaje más alto en $1^{\circ}$ año $(134,5)$ y el menor en $6^{\circ}$ año $(102,2)$.

\section{Asociación con años académicos}

Se encontró una asociación entre los años académicos y los puntajes obtenidos en el DREEM (escala y subescalas), ajustando por edad, estado académico y sexo (Tabla 3). Se observaron menores puntajes en los años académicos más avanzados $(<0,001)$. Consecuentemente, también se encontró una asociación negativa entre ser un estudiante de la etapa clínica y puntaje DREEM (escala y subescalas) en modelos multivariables ajustados por edad, estado académico y sexo (Tabla 4).

\section{Análisis de items}

En toda la muestra, seis ítems $(3,4,12,17$, 25 y 48) obtuvieron puntajes menores a 2,0, que significa "áreas problema" (Tabla 5). Sin embargo, tomando en cuenta solo la etapa clínica, se añaden 
Tabla 1. Medias del puntaje DREEM por subescalas del total de la muestra

\begin{tabular}{|c|c|c|c|}
\hline DREEM subescalas & Interpretación & Media (DE) & Ítems \\
\hline Percepción del aprendizaje & $\begin{array}{l}\text { 0-12 Muy pobre } \\
\text { 13-24 Es percibida negativamente } \\
\text { 25-36 Una percepción más positiva } \\
\text { 37-48 Es muy bien evaluada }\end{array}$ & $26,6(8,2)$ & 12 \\
\hline Percepción de los docentes & $\begin{array}{l}\text { 0-11 Abismal } \\
\text { 12-22 Necesitan entrenamiento educativo } \\
\text { 23-33 Encaminados en la dirección correcta } \\
\text { 34-44 Docentes modelo }\end{array}$ & $27,6 \quad(6,1)$ & 11 \\
\hline Autopercepción académica & $\begin{array}{l}\text { 0-8 Sentimientos de fracaso total } \\
\text { 9-16 Muchos aspectos negativos } \\
\text { 17-24 Sintiéndose más en el lado positivo } \\
\text { 25-32 Seguro del futuro académico }\end{array}$ & $21,0 \quad(4,5)$ & 8 \\
\hline Percepción de la atmósfera & $\begin{array}{l}\text { 0-12 Un ambiente pésimo } \\
\text { 13-24 Hay muchos aspectos que necesitan cambiar } \\
\text { 25-36 Una actitud más bien positiva } \\
\text { 37-48 Percepción general buena }\end{array}$ & $26,6 \quad(6,7)$ & 12 \\
\hline Autopercepción social & $\begin{array}{l}\text { 0-7 Miserable } \\
\text { 8-14 No es un buen lugar } \\
\text { 15-21 No tan mal ambiente social } \\
\text { 22-28 Muy buen ambiente social }\end{array}$ & $15,6 \quad(4,1)$ & 7 \\
\hline Total ambiente educativo & $\begin{array}{l}\text { 0-50 Muy pobre } \\
51-100 \text { Muchos problemas } \\
101-150 \text { Más positivo que negativo } \\
151-200 \text { Excelente }\end{array}$ & $117,2(25,6)$ & 50 \\
\hline
\end{tabular}

DE: Desviación Estándar.

Tabla 2. Características básicas de la muestra

\begin{tabular}{|lccccc|}
\hline $\begin{array}{l}\text { Etapa } \\
\text { académica }\end{array}$ & Año de estudio & $\begin{array}{c}\text { Respuestas / } \\
\text { Total de } \\
\text { alumnos (\%) }\end{array}$ & $\begin{array}{c}\text { M/F (Razón } \\
\text { M/F) }\end{array}$ & $\begin{array}{c}\text { Edad en años } \\
\text { Media (DE) }\end{array}$ & $\begin{array}{c}\text { Matrícula no } \\
\text { regular } \\
\mathbf{n}(\%)\end{array}$ \\
\hline Preclínica & $1^{\circ}$ & $173 / 554(31,2)$ & $72 / 101(0,7)$ & $17,9(1,8)$ & $10(5,8)$ \\
& $2^{\circ}$ & $167 / 401(41,7)$ & $63 / 104(0,6)$ & $19,0(1,5)$ & $43(25,8)$ \\
& $3^{\circ}$ & $117 / 486(24,1)$ & $54 / 63(0,8)$ & $20,6(1,5)$ & $51(43,6)$ \\
Clínica & $4^{\circ}$ & $136 / 342(39,8)$ & $51 / 85(0,6)$ & $21,7(1,9)$ & $14(10,3)$ \\
& $5^{\circ}$ & $137 / 309(44,3)$ & $53 / 84(0,6)$ & $22,6(1,7)$ & $1(0,7)$ \\
\hline Total & $6^{\circ}$ & $98 / 329(29,8)$ & $46 / 52(0,9)$ & $23,4(1,6)$ & $2(2,1)$ \\
\hline
\end{tabular}

M: Número de personas de sexo masculino; F: Número de personas de sexo femenino. DE: Desviación Estándar.

10 ítems con bajos puntajes $(13,14,20,21,24,41$, $42,43,44$ y 47). Además, se encontraron diferencias $(p<0,05)$ en los puntajes de los ítems entre la etapa preclínica y clínica (Tabla 5). Los ítems con menores puntajes fueron el ítem 3 y el 17, los cuales corresponden a "En la facultad, la copia en los exámenes constituye un problema" y "hay un buen sistema de apoyo para estudiantes que sufren estrés", respectivamente. No hubo ítems que sobrepasaron 3,50. Sin embargo, los ítems que obtuvieron los puntajes más altos fueron los ítems 10, 15 y 31 (Tabla 5). 
Tabla 3. Asociación entre el puntaje DREEM y los años académicos: modelo crudo y ajustado de regresión lineal

\begin{tabular}{|c|c|c|c|}
\hline Año académico & $\begin{array}{c}\text { Modelo crudo } \\
\text { Coeficiente- } \beta \text { (95\% IC) }\end{array}$ & $\begin{array}{l}\text { DREEM escala/subescalas } \\
\text { Modelo ajustado } \\
\text { Coeficiente- } \beta \text { ( } 95 \% \text { IC) }\end{array}$ & Media (DE) \\
\hline \multicolumn{4}{|c|}{ Percepción de aprendizaje (Rango de sub-escala:0-48) } \\
\hline $1^{\circ}$ & 1 (Referencia) & 1 (Referencia) & $32,5 \quad(6,5)$ \\
\hline $2^{\circ}$ & $-2,8(-4,3 ;-1,4)$ & $-2,7(-4,2 ;-1,2)$ & $29,7 \quad(5,3)$ \\
\hline $3^{\circ}$ & $-4,4(-6,0 ;-2,8)$ & $-4,1(-6,0 ;-2,3)$ & $28,2 \quad(6,2)$ \\
\hline $4^{\circ}$ & $-10,6(-12,1 ;-9,1)$ & $-10,5(-12,4 ;-8,7)$ & $22,0 \quad(8,2)^{*}$ \\
\hline $5^{\circ}$ & $-10,2(-11,7 ;-8,7)$ & $-10,2(-12,3 ;-8,2)$ & $22,3(7,2)^{*}$ \\
\hline $6^{\circ}$ & $-11,9(-13,6 ;-10,2)$ & $-11,9(-14,2 ;-9,7)$ & $20,6(7,7)^{*}$ \\
\hline \multicolumn{4}{|c|}{ Percepción de los docentes (Rango de subescala: 0 -44) } \\
\hline $1^{\circ}$ & 1 (Referencia) & 1 (Referencia) & $30,2 \quad(6,0)$ \\
\hline $2^{\circ}$ & $-2,6(-3,8 ;-1,3)$ & $-2,5(-3,8 ;-1,2)$ & $27,7 \quad(5,3)$ \\
\hline $3^{\circ}$ & $-2,9(-4,3 ;-1,5)$ & $-2,7(-4,3 ;-1,1)$ & $27,4 \quad(5,5)$ \\
\hline $4^{\circ}$ & $-3,3(-4,6 ;-2,0)$ & $-3,1(-4,7 ;-1,5)$ & $26,9 \quad(6,2)$ \\
\hline $5^{\circ}$ & $-4,9(-6,2 ;-3,6)$ & $-4,6(-6,4 ;-2,9)$ & $25,4 \quad(6,4)$ \\
\hline $6^{\circ}$ & $-3,9(-5,4 ;-2,5)$ & $-3,7(-5,6 ;-1,7)$ & $26,3 \quad(6,2)$ \\
\hline \multicolumn{4}{|c|}{ Autopercepción académica (Rango de subescala: 0 -32) } \\
\hline $1^{\circ}$ & 1 (Referencia) & 1 (Referencia) & $22,9 \quad(4,7)$ \\
\hline $2^{\circ}$ & $-0,7(-1,7 ; 0,2)$ & $-0,6(-1,6 ; 0,3)$ & $22,1 \quad(3,7)$ \\
\hline $3^{\circ}$ & $-1,5(-2,5 ;-0,4)$ & $-1,4(-2,5 ;-0,3)$ & $21,4 \quad(3,5)$ \\
\hline $4^{\circ}$ & $-3,2(-4,2 ;-2,2)$ & $-3,4(-4,6 ;-2,3)$ & $19,7 \quad(4,7)$ \\
\hline $5^{\circ}$ & $-3,2(-4,2 ;-2,2)$ & $-3,7(-4,9 ;-2,4)$ & $19,7 \quad(4,5)$ \\
\hline $6^{\circ}$ & $-3,9(-4,9 ;-2,8)$ & $-4,5(-5,9 ;-3,0)$ & $19,0 \quad(4,5)$ \\
\hline \multicolumn{4}{|c|}{ Percepción de la atmósfera (Rango de subescala: $0-48$ ) } \\
\hline $1^{\circ}$ & 1 (Referencia) & 1 (Referencia) & $31,0 \quad(6,5)$ \\
\hline $2^{\circ}$ & $-2,9(-4,2 ;-1,6)$ & $-2,7(-4,0-1,3)$ & $28,1 \quad(5,4)$ \\
\hline $3^{\circ}$ & $-4,1(-5,5 ;-2,7)$ & $-3,8(-5,4,-2,3)$ & $26,9 \quad(5,3)$ \\
\hline $4^{\circ}$ & $-6,7(-8,0 ;-5,3)$ & $-6,9(-8,5,-5,3)$ & $24,3(6,2)^{*}$ \\
\hline $5^{\circ}$ & $-6,7(-8,0 ;-5,3)$ & $-7,1(-8,9,-5,3)$ & $24,3(6,5)^{*}$ \\
\hline $6^{\circ}$ & $-8,5(-10,0 ;-7,0)$ & $-9,1(-11,1,-7,1)$ & $22,5(6,3)^{*}$ \\
\hline \multicolumn{4}{|c|}{ Autopercepción social (Rango de subescala: 0-28) } \\
\hline $1^{\circ}$ & 1 (Referencia) & 1 (Referencia) & $17,9 \quad(4,3)$ \\
\hline $2^{\circ}$ & $-1,4(-2,3 ;-0,6)$ & $-1,4(-2,2 ;-0,5)$ & $16,5 \quad(3,4)$ \\
\hline $3^{\circ}$ & $-2,5(-3,4 ;-1,6)$ & $-2,4(-3,5 ;-1,4)$ & $15,4 \quad(3,6)$ \\
\hline $4^{\circ}$ & $-4,0(-4,8 ;-3,1)$ & $-4,0(-5,0 ;-2,9)$ & $14,0(4,1)^{*}$ \\
\hline $5^{\circ}$ & $-3,2(-4,1 ;-2,4)$ & $-3,3(-4,4 ;-2,1)$ & $14,7 \quad(4,0)^{*}$ \\
\hline $6^{\circ}$ & $-4,2(-5,1 ;-3,2)$ & $-4,3(-5,6 ;-3,0)$ & $13,8 \quad(3,6)^{*}$ \\
\hline \multicolumn{4}{|c|}{ Puntaje total (Rango de subescala: $0-200$ ) } \\
\hline $1^{\circ}$ & 1 (Referencia) & 1 (Referencia) & $134,5(24,2)$ \\
\hline $2^{\circ}$ & $-10,5(-15,4 ;-5,6)$ & $-9,8(-14,8 ;-4,8)$ & $124,0(19,3)$ \\
\hline $3^{\circ}$ & $-15,3(-20,7 ;-9,9)$ & $-14,5(-20,5 ;-8,4)$ & $119,2(19,6)$ \\
\hline $4^{\circ}$ & $-27,7(-32,8 ;-22,5)$ & $-27,9(34,1 ;-21,7)$ & $106,9(25,2)$ \\
\hline $5^{\circ}$ & $-28,1(-33,3 ;-23,0)$ & $-28,9(-35,7 ;-22,1)$ & $106,4(24,6)$ \\
\hline $6^{\circ}$ & $-32,7(-38,0 ;-26,7)$ & $-33,5(-41,1 ;-25,8)$ & $102,2(24,1)$ \\
\hline
\end{tabular}

a Modelo fue ajustado por sexo, edad y condición de matrícula; * Indica puntajes considerados negativos de acuerdo a la interpretación por sub-escalas. 
Tabla 4. Puntaje DREEM y etapas académicas: modelo crudo y ajustado de regresión lineal

\begin{tabular}{|c|c|c|c|c|c|}
\hline $\begin{array}{c}\text { DREEM } \\
\text { Subescalas }\end{array}$ & $\begin{array}{l}\text { Preclínica } \\
\text { Media (DE) }\end{array}$ & $\begin{array}{c}\text { Clínica } \\
\text { Media (DE) }\end{array}$ & Valor de $p$ & $\begin{array}{l}\text { Modelo crudo } \\
\text { Coeficiente- } \beta \\
(95 \% \text { IC) }\end{array}$ & $\begin{array}{c}\text { Modelo ajustado } \\
\text { Coeficiente- } \beta \\
(95 \% \text { IC })\end{array}$ \\
\hline Aprendizaje & $30,4 \quad(6,2)$ & $21,7 \quad(7,7)^{* *}$ & $<0,001$ & $-8,6 \quad(-9,6 ;-7,7)$ & $-8,0 \quad(-9,4 ;-6,7)$ \\
\hline Docentes & $28,6 \quad(5,7)$ & $26,2 \quad(6,3)$ & $<0,001$ & $-2,4 \quad(-3,2 ;-1,6)$ & $-1,7 \quad(-2,9 ;-0,5)$ \\
\hline Académico & $22,2 \quad(4,2)$ & $19,4 \quad(4,6)$ & $<0,001$ & $-2,7 \quad(-3,3 ;-2,1)$ & $-2,9 \quad(-3,7 ;-2,0)$ \\
\hline Atmósfera & $28,9 \quad(6,0)$ & $23,8 \quad(6,3)^{* *}$ & $<0,001$ & $-5,0 \quad(-5,9 ;-4,2)$ & $-4,8 \quad(-6,0 ;-3,6)$ \\
\hline Social & $16,7 \quad(3,9)$ & $14,2 \quad(3,9)^{* *}$ & $<0,001$ & $-2,6 \quad(-3,2 ;-2,0)$ & $-2,3 \quad(-3,2 ;-1,6)$ \\
\hline Total & $126,8(22,2)$ & $105,4(24,7)$ & $<0,001$ & $-21,3(-24,5 ;-18,1)$ & $-19,8(-24,3 ;-15,2)$ \\
\hline
\end{tabular}

aModelo ha sido ajustado por edad, sexo y condición de matrícula. La referencia en los modelos es el puntaje en área preclínica. Se utilizó la prueba T-prueba para comparar entre puntaje preclínica y clínica; **Puntajes considerados más negativos que positivos. DE: Desviación Estándar.

Tabla 5. Promedios del puntaje DREEM por ítems en etapa preclínica, clínica y la muestra total

\begin{tabular}{|c|c|c|c|c|}
\hline Ítems & $\begin{array}{l}\text { Media total } \\
\text { (DE) }\end{array}$ & $\begin{array}{l}\text { Preclínica } \\
\text { Media (DE) }\end{array}$ & $\begin{array}{l}\text { Clínica } \\
\text { Media (DE) }\end{array}$ & $\begin{array}{c}\text { Valor } \\
p\end{array}$ \\
\hline \multicolumn{5}{|l|}{ Percepción de aprendizaje } \\
\hline 1 Se me estimula a participar en clase & $2,6(1,0)$ & $3,0(0,8)$ & $2,2(1,0)$ & $<0,001$ \\
\hline 7 La enseñanza es frecuentemente estimulante & $2,4(1,0)$ & $2,6(0,9)$ & $2,0(1,1)$ & $<0,001$ \\
\hline 13 La enseñanza es centrada en el estudiante** & $2,2(1,1)$ & $2,6(0,4)$ & $1,7(0,6)$ & $<0,001$ \\
\hline $\begin{array}{l}16 \text { La enseñanza que se me brinda me ayuda a desarrollar } \\
\text { mis competencias }\end{array}$ & $2,6(0,9)$ & $2,9(0,7)$ & $2,3(1,0)$ & $<0,001$ \\
\hline 20 La enseñanza está bien enfocada** & $2,3(1,0)$ & $2,7(0,9)$ & $1,7(1,1)$ & $<0,001$ \\
\hline $\begin{array}{l}21 \text { La enseñanza en la facultad está suficientemente preo- } \\
\text { cupada de desarrollar mi confianza** }\end{array}$ & $2,0(1,1)$ & $2,4(1,0)$ & $1,5(0,9)$ & $<0,001$ \\
\hline 24 El tiempo destinado a la docencia es bien utilizado** & $2,3(1,0)$ & $2,6(0,9)$ & $1,8(1,0)$ & $<0,001$ \\
\hline $\begin{array}{l}25 \text { La enseñanza en la facultad pone demasiado énfasis en } \\
\text { el aprendizaje de detalles innecesarios }\end{array}$ & $1,6(1,1)$ & $1,8(1,0)$ & $1,4(1,0)$ & $<0,001$ \\
\hline 38 Tengo claros los objetivos de aprendizaje de mis cursos & $2,6(0,9)$ & $2,8(0,8)$ & $2,4(1,0)$ & $<0,001$ \\
\hline $\begin{array}{l}44 \text { La manera de enseñar me estimula a aprender por mí } \\
\text { mismo en forma activa** }\end{array}$ & $2,3(1,1)$ & $2,7(0,9)$ & $1,8(1,1)$ & $<0,001$ \\
\hline $\begin{array}{l}47 \text { En la facultad, se enfatiza el aprendizaje a largo plazo } \\
\text { por sobre el inmediato** }\end{array}$ & $2,0(1,2)$ & $2,5(1,0)$ & $1,4(1,1)$ & $<0,001$ \\
\hline $\begin{array}{l}48 \text { La enseñanza de la facultad está demasiado centrada } \\
\text { en los docentes }\end{array}$ & $1,7(0,9)$ & $1,9(0,9)$ & $1,5(0,9)$ & $<0,001$ \\
\hline
\end{tabular}


Tabla 5. Continuación

\begin{tabular}{|c|c|c|c|c|}
\hline ítems & $\begin{array}{l}\text { Media total } \\
\text { (DE) }\end{array}$ & $\begin{array}{l}\text { Preclínica } \\
\text { Media (DE) }\end{array}$ & $\begin{array}{c}\text { Clínica } \\
\text { Media (DE) }\end{array}$ & $\begin{array}{l}\text { Valor } \\
\mathbf{p}\end{array}$ \\
\hline \multicolumn{5}{|l|}{ Percepción de los docentes } \\
\hline 2 Los docentes conocen las materias que dictan & $2,9(0,7)$ & $3,1(0,7)$ & $2,8(0,7)$ & $<0,001$ \\
\hline 6 Los docentes tienen paciencia con los pacientes & $2,6(0,9)$ & $2,6(0,9)$ & $2,6(0,9)$ & 0,71 \\
\hline 8 Los docentes ridiculizan a los estudiantes & $2,4(1,1)$ & $2,5(1,1)$ & $2,2(1,1)$ & $<0,001$ \\
\hline 9 Los docentes son autoritarios & $2,1(1,1)$ & $2,1(1,1)$ & $2,1(1,1)$ & 0,96 \\
\hline $\begin{array}{l}18 \text { Los docentes tienen buenas destrezas comunicacionales } \\
\text { con los pacientes }\end{array}$ & $2,7(0,8)$ & $2,7(0,8)$ & $2,7(0,8)$ & 0,99 \\
\hline $\begin{array}{l}29 \text { Los docentes son buenos dando feedback (retroalimen- } \\
\text { tación) a los estudiantes }\end{array}$ & $2,3(1,0)$ & $2,5(0,9)$ & $2,1(1,0)$ & $<0,001$ \\
\hline $\begin{array}{l}32 \text { En la facultad, los profesores nos hacen críticas cons- } \\
\text { tructivas }\end{array}$ & $2,7(0,9)$ & $2,9(0,8)$ & $2,4(1,0)$ & $<0,001$ \\
\hline 37 Los docentes dan ejemplos claros & $2,5(0,9)$ & $2,7(0,8)$ & $2,3(0,9)$ & $<0,001$ \\
\hline 39 Los docentes se molestan y se alteran en clase & $2,5(1,0)$ & $2,5(1,1)$ & $2,4(0,9)$ & 0,39 \\
\hline 40 Los docentes están bien preparados para sus clases & $2,6(0,9)$ & $2,9(0,8)$ & $2,3(1,0)$ & $<0,001$ \\
\hline 49 Los estudiantes causamos irritación a los docentes & $2,3(1,0)$ & $2,2(1,0)$ & $2,4(1,0)$ & 0,06 \\
\hline \multicolumn{5}{|l|}{ Autopercepción académica } \\
\hline $\begin{array}{l}5 \text { Los métodos de estudio que tenía antes todavía me } \\
\text { sirven }\end{array}$ & $2,3(1,0)$ & $2,4(1,0)$ & $2,2(1,0)$ & 0,10 \\
\hline 10 Tengo la confianza de que voy a pasar este año & $3,1(1,0)$ & $3,1(0,9)$ & $3,0(1,0)$ & 0,48 \\
\hline $\begin{array}{l}22 \text { Siento que me están preparando bien para mi profe- } \\
\text { sión }\end{array}$ & $2,6(1,0)$ & $3,0(0,75)$ & $2,1(1,0)$ & $<0,001$ \\
\hline $\begin{array}{l}26 \text { Lo aprendido el año pasado fue una buena base para el } \\
\text { trabajo de este año }\end{array}$ & $2,7(0,9)$ & $2,8(0,8)$ & $2,6(0,9)$ & $<0,001$ \\
\hline 27 Soy capaz de memorizar todo lo que me es necesario & $2,3(1,0)$ & $2,5(1,0)$ & $2,1(1,1)$ & $<0,001$ \\
\hline 31 He aprendido mucho sobre la empatía en mi profesión & $3,1(0,8)$ & $3,2(0,7)$ & $3,0(0,9)$ & 0,001 \\
\hline $\begin{array}{l}41 \text { La facultad me ayuda a desarrollar mis destrezas para } \\
\text { resolver problemas** }\end{array}$ & $2,2(1,0)$ & $2,6(1,0)$ & $1,8(1,0)$ & $<0,001$ \\
\hline $\begin{array}{l}45 \text { Mucho de lo que tengo que aprender me parece rele- } \\
\text { vante para mi carrera como médico. }\end{array}$ & $2,8(0,9)$ & $2,8(0,9)$ & $2,7(0,9)$ & 0,12 \\
\hline \multicolumn{5}{|l|}{ Percepción de la atmósfera } \\
\hline $\begin{array}{l}11 \text { El ambiente es relajado durante las visitas docentes de } \\
\text { los servicios hospitalarios. }\end{array}$ & $2,5(0,9)$ & $2,5(0,8)$ & $2,4(1,0)$ & 0,04 \\
\hline 12 Los horarios de la facultad están bien programados & $1,5(1,2)$ & $1,8(1,2)$ & $1,0(1,2)$ & $<0,001$ \\
\hline $\begin{array}{l}17 \text { En la facultad, la copia en los exámenes constituye un } \\
\text { problema }\end{array}$ & $1,0(1,0)$ & $1,1(1,0)$ & $0,9(1,1)$ & $<0,05$ \\
\hline $\begin{array}{l}23 \text { El ambiente es tranquilo y relajado durante las clases } \\
\text { teóricas en el aula. }\end{array}$ & $2,4(1,0)$ & $2,7(0,9)$ & $2,1(1,1)$ & $<0,001$ \\
\hline $\begin{array}{l}30 \text { Tengo oportunidades para desarrollar mis habilidades } \\
\text { interpersonales }\end{array}$ & $2,4(1,1)$ & $2,6(1,0)$ & $2,1(1,1)$ & $<0,001$ \\
\hline 33 Me siento cómodo, socialmente, en clases & $2,8(0,9)$ & $2,8(0,9)$ & $2,7(0,9)$ & $<0,05$ \\
\hline
\end{tabular}


Tabla 5. Continuación

\begin{tabular}{|c|c|c|c|c|}
\hline ítems & $\begin{array}{l}\text { Media total } \\
\text { (DE) }\end{array}$ & $\begin{array}{l}\text { Preclínica } \\
\text { Media (DE) }\end{array}$ & $\begin{array}{l}\text { Clínica } \\
\text { Media (DE) }\end{array}$ & $\begin{array}{l}\text { Valor } \\
\mathbf{p}\end{array}$ \\
\hline $\begin{array}{l}34 \text { El ambiente en los seminarios, clases y prácticas es } \\
\text { tranquilo }\end{array}$ & $2,8(0,8)$ & $3,0(0,8)$ & $2,7(0,9)$ & $<0,001$ \\
\hline $\begin{array}{l}35 \text { Hasta el momento, mi experiencia en la facultad ha } \\
\text { sido desalentadora }\end{array}$ & $2,4(1,1)$ & $2,6(1,0)$ & $2,1(1,1)$ & $<0,001$ \\
\hline 36 En general, soy capaz de concentrarme bien. & $2,5(0,9)$ & $2,6(0,9)$ & $2,4(0,9)$ & $<0,05$ \\
\hline $\begin{array}{l}42 \text { El disfrute de mis estudios en la facultad pesa más que } \\
\text { la tensión que éstos me generan** }\end{array}$ & $2,2(1,1)$ & $2,4(1,0)$ & $1,7(1,1)$ & $<0,001$ \\
\hline 43 El ambiente de la facultad me motiva a aprender** & $2,1(1,1)$ & $2,5(1,0)$ & $1,7(1,0)$ & $<0,001$ \\
\hline 50 Siento que puedo hacer todas las preguntas que quiero & $2,2(1,0)$ & $2,4(1,0)$ & $2,0(1,1)$ & $<0,001$ \\
\hline \multicolumn{5}{|l|}{ Autopercepción social } \\
\hline $\begin{array}{l}3 \text { Hay un buen sistema de apoyo para los estudiantes que } \\
\text { sufren de estrés }\end{array}$ & $1,3(1,1)$ & $1,6(1,0)$ & $0,8(0,9)$ & $<0,001$ \\
\hline $\begin{array}{l}4 \text { Estoy demasiado cansado para disfrutar los cursos que } \\
\text { estoy tomando }\end{array}$ & $1,7(1,1)$ & $1,8(1,1)$ & $1,5(1,1)$ & $<0,001$ \\
\hline 14 Rara vez me aburro en los cursos que estoy tomando** & $2,0(1,1)$ & $2,3(1,0)$ & $1,7(1,1)$ & $<0,001$ \\
\hline 15 Tengo buenos amigos en la facultad & $3,3(0,8)$ & $3,3(0,8)$ & $3,3(0,8)$ & 0,85 \\
\hline 19 Mi vida social es buena & $2,6(1,0)$ & $2,8(0,9)$ & $2,4(1,1)$ & $<0,001$ \\
\hline 28 Rara vez me siento solo & $2,4(1,1)$ & $2,5(1,1)$ & $2,4(1,1)$ & 0,82 \\
\hline 46 Los ambientes físicos de la facultad son agradables & $2,4(1,1)$ & $2,5(1,0)$ & $2,2(1,1)$ & $<0,001$ \\
\hline
\end{tabular}

*'tems que tienen puntaje $<2,0$ en la etapa clínica y/o pre-clínica. Los ítems en cursiva están codificados negativamente. Sin embargo, sus puntajes están mostrados de forma revertida, después del análisis. A menor valor, peor desempeño en esas áreas. Se utilizó T-test para diferenciar puntajes entre etapa pre-clínica y clínica. DE: Desviación Estándar.

\section{Discusión}

El ambiente educativo de esta facultad de medicina obtuvo una puntuación correspondiente a "más aspectos positivos que negativos" en la escala DREEM. Sin embargo, se observó una tendencia negativa en la percepción del ambiente educativo a mayor año académico. Los principales problemas identificados se pueden resumir en cinco: enseñanza basada en el docente, horarios inadecuados, enseñanza que genera poco estímulo y disminución de la confianza, copia en los exámenes y la falta de apoyo en situaciones de estrés. Estos problemas, se acrecientan en la etapa clínica.

\section{Puntuación general}

En nuestra muestra, la escala DREEM mostró una puntuación positiva similar a la encontrada en otras escuelas de medicina latinoamericanas ${ }^{2,11}$. En un estudio realizado en seis escuelas de medicina en Chile, que incluyó solamente a estudiantes de $3^{\circ}, 4^{\circ}$ y $5^{\circ}$ año académico, las puntuaciones totales de DREEM por facultad oscilaron entre 103,1 y 126,9 puntos $^{2}$. De similar manera, en una facultad de medicina argentina, los puntajes totales de DREEM de $1^{\circ}, 3^{\circ}$ y $5^{\circ}$ año académico fueron 149,6 , 142,4 y 136,6 , respectivamente. Este estudio, que presentó puntajes más altos que el nuestro, sugiere que estos se debían a su nuevo plan de estudios basado en problemas ${ }^{11}$.

\section{Tendencia negativa}

Nuestro estudio mostró una tendencia negativa en los puntajes DREEM en años superiores del programa de pregrado. De forma similar, algunos estudios ${ }^{11,13,21}$ han encontrado puntajes negativos en años académicos mayores, aunque tomando como referencia solo un año por etapa 
académica y no el total de años, como nuestro estudio. De manera general, esta tendencia negativa puede deberse a un aumento en el estrés en estudiantes en los años académicos superiores ${ }^{21} \mathrm{o}$ que los estudiantes de grados menores no tienen experiencia suficiente para hacer una evaluación adecuada $^{22}$. De las subescalas, la percepción social de aprendizaje, de atmósfera y la autopercepción resultaron ser las más débiles. Sin embargo, es el análisis de ítems que nos ha permitido identificar 5 principales problemas, más evidentes en el área clínica:

Primero, la enseñanza basada en el docente (ítem 48) y el aprendizaje de datos innecesarios (ítem 25). La memorización de información innecesaria es un reclamo común en las escuelas de medicina de América Latina, Asia y Europa ${ }^{2,23,24}$. Por otro lado, el enfoque basado en docente es tradicional, compuesto principalmente por la transmisión de información por parte del profesor, dejando al alumno como un ente pasivo ${ }^{25}$.

Segundo, los horarios no son bien programados (ítem 12) y el tiempo de enseñanza no es bien utilizado (ítem 24). En la etapa clínica, el tiempo de enseñanza en los hospitales puede ser limitado debido a las responsabilidades del personal médico en los hospitales. Este problema también se ha descrito en España, Alemania y el Reino Unido ${ }^{23,24,27}$. Además, existe una gran cantidad de estudiantes por grupo de práctica hospitalaria, lo cual puede afectar la satisfacción del alumno ${ }^{28}$.

Tercero, en la etapa clínica, la enseñanza parece no generar un estímulo por aprender ni generar confianza (ítem 21, 41, 43 y 44). Por ello, la metodología de enseñanza en los ambientes hospitalarios, en combinación con las clases teóricas brindadas, debe ser evaluada y replanteadas.

Cuarto, toda la facultad menciona que copiar en los exámenes es un problema importante (ítem 17). Esta mala conducta académica ha sido descrita frecuentemente en América Latina y Europa ${ }^{2,21,23,26}$. El problema dentro de la profesión médica parece ser impulsado por la presión para tener éxito, la falta de comprensión de lo que constituye copiar y el medio ambiente que fomenta comportamiento deshonesto ${ }^{29}$.

Quinto, los estudiantes perciben que existe un excesivo cansancio (ítem 4) y que no hay un buen sistema de apoyo para los que sufren estrés (ítem 3 ). Aunque la facultad de medicina tiene una unidad de apoyo psicológico y tutoría, puede ser que no se de abasto (tiempo y recursos humanos) con brindar apoyo a estos estudiantes o peor aun, que sean alumnos que aún no han sido identificados o no sepan la existencia de este servicio. Además, estudiantes de clínica refieren que la tensión es mayor al disfrute de sus estudios (ítem 42) y que les genera aburrimiento (ítem 14).

Por otro lado, es importante que los estudiantes desarrollan empatía durante su carrera.

\section{Relevancia o implicaciones}

Este estudio tiene implicaciones en la educación médica. En primer lugar, las áreas problemas expuestas deben ser prioridad por las autoridades de la universidad. Además, el estudio alienta a otras facultades a que planteen sus propios estudios y evalúen su ambiente educativo. Finalmente, la SUNEDU y el Ministerio de Educación deben considerar los resultados del estudio para plantear evaluaciones periódicas y soluciones, principalmente respecto a las conductas éticas (plagio) y el apoyo a los estudiantes en situaciones de estrés. Por otro lado, con las áreas problema identificadas, se pueden diseñar estudios cualitativos para ahondar en las explicaciones y soluciones.

\section{Fortalezas y limitaciones}

Una fortaleza de este estudio es que ha explorado la percepción del ambiente educativo de 6 años académicos. Además, aunque un estudio peruano utilizó el DREEM para evaluar una rotación hospitalaria $^{30}$, este es el primer estudio que lo utiliza para evaluar globalmente la totalidad de las etapas preclínica y clínica. Sin embargo, este estudio también presenta algunas limitaciones. Usualmente, los cuestionarios virtuales tienen menor tasa de respuesta que los físico ${ }^{31}, y$, quizás, los alumnos que respondieron el cuestionario eran aquellos que deseaban expresar su descontento (sesgo de deseabilidad). Sin embargo, nuestra intención de comparar los puntajes por año académico, disminuye esta limitación. Por otro lado, en el análisis ajustado, puede haber confusores que no se evaluaron, como el tiempo de demora en llegar a la facultad, su estabilidad económica, entre otras.

\section{Conclusiones}

El ambiente educativo percibido por los estudiantes de esta facultad de medicina resultó tener un puntaje positivo. Sin embargo, se evidenció 
una tendencia negativa, con puntajes menores en alumnos de mayor grado académico o etapa clínica. Los puntos críticos del ambiente educativo parecen estar relacionados con una enseñanza basada en el docente, la inadecuada programación de horarios, el poco estímulo para generar confianza y aprendizaje, la copia en los exámenes, y falta de soporte en situaciones de estrés.

\section{Referencias}

1. Olave G, Pérez C, Fasce E, Ortiz L, Bastías N, Márquez $\mathrm{C}$, et al. Factores que afectan al ambiente educativo en la formación preclínica de medicina según sus docentes. Rev Med Chile 2016; 144(10): 1343-50.

2. Herrera C, Pacheco J, Rosso F, Cisterna C, Aichele D, Becker S, et al. Evaluación del ambiente educacional pre-clínico en seis Escuelas de Medicina en Chile. Rev Med Chile 2010; 138(6): 677-84.

3. American Medical Association. Initiative to Transform Medical Education. Strategies for transforming the medical education learning environment. Phase 3: Program implementation. Final report of the December. 2008. Disponible en: https://www.gmc-uk.org/Educational_Environments__May_2013.pdf_52096709.pdf

4. World Federation for Medical Education. International standards in medical education: assessment and accreditation of medical schools' - educational programmes: a WFME position paper. Med Educ 1998; 32: 549-58.

5. Saarikoski M, Leino-Kilpi H. Association between quality of ward nursing care and students' assessment of the ward as a learning environment. NT Research 1999; 4(6): 467-74.

6. Pace CR, Stern GG. An approach to the measurement of psychological characteristics of college environments. J Educ Psychol 1958; 49(5): 269-77.

7. Tackett S, Shochet R, Shilkofski NA, Colbert-Getz J, Rampal K, Abu Bakar H, et al. Learning environment assessments of a single curriculum being taught at two medical schools 10,000 miles apart. BMC Med Educ 2015; 15: 105.

8. Miles S, Swift L, Leinster SJ. The Dundee Ready Education Environment Measure (DREEM): a review of its adoption and use. Med Teach 2012; 34(9): e620-34.

9. Genn JM. AMEE Medical Education Guide No. 23 (Part 2): Curriculum, environment, climate, quality and change in medical education - a unifying perspective. Med Teach 2001; 23(5): 445-54.

10. Roff S. The Dundee Ready Educational Environment Measure (DREEM)-a generic instrument for measuring students' perceptions of undergraduate health professions curricula. Med Teach 2005; 27(4): 322-5.

11. Díaz-Véliz G, Mora S, Bianchi R, Gargiulo PA, Terán C, Gorena D, et al. Percepción de los estudiantes de medicina del ambiente educativo en una facultad con currículo tradicional (UCH-Chile) y otra con currículo basado en problemas (UNC-Argentina). Educ Med 2011; 14(1): 27-34.

12. Riquelme A, Oporto M, Oporto J, Mendez JI, Viviani $\mathrm{P}$, Salech F, et al. Measuring students' perceptions of the educational climate of the new curriculum at the Pontificia Universidad Católica de Chile: performance of the Spanish translation of the Dundee Ready Education Environment Measure (DREEM). Educ Health (Abingdon, England) 2009; 22(1): 112.

13. Díaz-Véliz G, Mora S, Escanero JF. Percepción del ambiente educacional en dos escuelas de medicina con currículo tradicional: Estudio longitudinal. Rev Med Chile 2016; 144(11): 1479-85.

14. Diario Gestión. Comisión del Minsa pide detener creación de escuelas o facultades de medicina en Perú. 2017 Disponible en: https://gestion.pe/tendencias/management-empleo/comision-minsa-pide-detener-creacion-escuelas-facultades-medicina-peru-129579)

15. Diario El Peruano. Ley Universitaria 30220-2014. 2014. Disponible en: https://www.sunedu.gob.pe/nueva-ley-universitaria-30220-2014/

16. Universidad de San Martín de Porres. Reglamento de evaluación de estudiantes de pregrado. 2015. Disponible en: http://www.medicina.usmp.edu.pe/medicina/ academico/normativas/REGLAMENTO_DE_ESTUDIANTES_2015.pdf

17. McAleer S, Roff S. A practical guide to using the Dundee Ready Education Environment Measure (DREEM). AMEE Guides 2001; 23: 29-33.

18. Hammond SM, O'Rourke M, Kelly M, Bennett D, O'Flynn S. A psychometric appraisal of the DREEM. BMC Med Educ 2012; 12: 2.

19. Ortega J, Pérez C, Ortiz L, Fasce E, McColl P, Torres A, et al. [An assessment of the Dundee Ready Education Environment Measure (DREEM) in Chilean university students]. Rev Med Chile 2015; 143(5): 651-7.

20. Flores-Flores O, Lajo-Aurazo Y, Zevallos-Morales A, Rondán PL, Lizaraso-Soto F, Jorquiera T. Análisis psicométrico de un cuestionario para medir el ambiente educativo en una muestra de estudiantes de medicina en Perú. Rev Peru Med Exp Salud Publica 2017; 34(2): 225-60.

21. Hasan T, Gupta P. Assessing the learning environment at Jazan Medical School of Saudi Arabia. Med Teach 2013; 35(S1): S90-6. 
22. Taheri M. Students' perceptions of learning environment in Guilan University of Medical Sciences. J Med Educ 2013; 13(4).

23. Pales J, Gual A, Escaneroi J, Tomás I, Rodríguez de Castro F, Elorudy M, et al. Educational climate perception by preclinical and clinical medical students in five Spanish medical schools. Int J Med Educ 2015; 6: 65-75.

24. Jiffry M, McAleer S, Fernando S, Marasinghe R. Using the DREEM questionnaire to gather baseline information on an evolving medical school in Sri Lanka. Med Teach 2005; 27(4): 348-52.

25. Elen J, Clarebout G, Léonard R, Lowyck J. Student-centred and teacher-centred learning environments: what students think. Teach High Educ 2007; 12(1): 105-17.

26. Rotthoff T, Ostapczuk MS, De Bruin J, Decking U, Schneider M, Ritz-Timme S. Assessing the learning environment of a faculty: psychometric validation of the German version of the Dundee Ready Education Environment Measure with students and teachers. Med Teach 2011; 33(11): e624-36.
27. Hendry RG, Kawai GK, Moody WE, Sheppard JE, Smith LC, Richardson M, et al. Consultant attitudes to undertaking undergraduate teaching duties: perspectives from hospitals serving a large medical school. Med Educ 2005; 39(11): 1129-39.

28. Condon B, Worley P, Condon J, Prideaux D. Student academic performance in rural clinical schools: The impact of cohort size and competition. Med Teach 2017; 39(3): 262-8.

29. Kusnoor AV, Falik R. Cheating in medical school: the unacknowledged ailment. South Med J 2013; 106(8): 479-83.

30. San-Martín M, Rivera EM, Alcorta-Garza A, Vivanco L. Moral perception, educational environment, and development of medical professionalism in medical students during the clinical rotations in Peru. International Journal of Ethics Education 2016; 1(2): 163-72.

31. Nulty DD. The Adequacy of Response Rates to Online and Paper Surveys: What Can Be Done? Assess Eval High Educ 2008; 33(3): 301-14. 